\title{
BMJ Open Risk of pneumothorax in pneumoconiosis patients in Taiwan: a retrospective cohort study
}

\author{
Jo-Hui Pan, ${ }^{1,2}$ Chih-Hung Cheng, ${ }^{2,3}$ Chao-Ling Wang, ${ }^{1}$ Chia-Yen Dai, ${ }^{1,4}$ \\ Chau-Chyun Sheu (D) , 3,4,5 Ming-Ju Tsai (D) , ,4,5 Jen-Yu Hung, ${ }^{2,3,4,5,6}$ \\ Inn-Wen Chong ${ }^{3,4,5}$
}

To cite: Pan J-H, Cheng C$\mathrm{H}$, Wang C-L, et al. Risk of pneumothorax in pneumoconiosis patients in Taiwan: a retrospective cohort study. BMJ Open 2021;11:e054098. doi:10.1136/ bmjopen-2021-054098

- Prepublication history and additional supplemental material for this paper are available online. To view these files, please visit the journal online (http://dx.doi.org/10.1136/ bmjopen-2021-054098).

M-JT and J-YH contributed equally.

Received 02 June 2021 Accepted 19 September 2021

\section{ABSTRACT}

Objectives This study was conducted to explore the association between pneumoconiosis and pneumothorax. Design Retrospective cohort study.

Setting Nationwide population-based study using the Taiwan National Health Insurance Database.

Participants A total of 2333 pneumoconiosis patients were identified (1935 patients for propensity score (PS)matched cohort) and matched to 23330 control subjects by age and sex (7740 subjects for PS-matched cohort). Primary and secondary outcome measures The incidence and the cumulative incidence of pneumothorax. Results Both incidence and the cumulative incidence of pneumothorax were significantly higher in the pneumoconiosis patients as compared with the control subjects $(p<0.0001)$. For multivariable Cox regression analysis adjusted for age, sex, residency, income level and other comorbidities, patients with pneumoconiosis exhibited a significantly higher risk of pneumothorax than those without pneumoconiosis (HR 3.05, 95\% Cl 2.18 to $4.28, p<0.0001)$. The male sex, heart disease, peripheral vascular disease, chronic pulmonary disease and connective tissue disease were risk factors for developing pneumothorax in pneumoconiosis patients.

Conclusions Our study revealed a higher risk of pneumothorax in pneumoconiosis patients and suggested potential risk factors in these patients. Clinicians should be aware about the risk of pneumothorax in pneumoconiosis patients.

\section{INTRODUCTION}

Pneumoconiosis, one of the oldest occupational diseases, continues to disable the health of labourers to this day. Three kinds of dusts, including coal dust, crystalline silica and asbestos, are mainly responsible for this disease ${ }^{1}$ with the deposition of this (inhaled) inorganic dust causing irreversible fibrotic reaction in lung tissue through inflammatory cascades. Most patients develop respiratory symptoms, lung function impairment and chest radiologic abnormalities, and the disease may progress even after cessation of exposure. $^{23}$

\section{Strengths and limitations of this study}

- The nationwide population-based data could minimise the selection bias with a broad view of the real-world epidemiology.

- This long-term cohort study provides better evidence about the temporal trajectory for the association between pneumoconiosis and pneumothorax.

- The propensity-scored-matched model was applied to account for confounding factors.

- The risk factors for incident pneumothorax in pneumoconiosis patients were also disclosed in our study.

- This study was limited by the shortage of clinical information, such as smoking history, in the National Health Insurance database.

Pneumoconiosis, maintaining a relatively high incidence in recent years, is still prevalent worldwide. ${ }^{4}$ Although it is thought to be preventable, endemic resurgence is still reported, even in developed countries. ${ }^{5} 6$ According to the Global Burden of Disease study of 2017, approximately 528000 cases were living with pneumoconiosis, ${ }^{7}$ and 21 600 cases of global deaths were resulted from pneumoconiosis. ${ }^{8}$ Despite of regulation control and health surveillance, medical care for pneumoconiosis patients is unneglectable. However, with limited treatment options for pneumoconiosis, management has focused merely on alleviating progression of the disease as well as the complications or comorbidities.

Pneumothorax has been considered as one of the major complications of pneumoconiosis as reported in different countries and industries. ${ }^{4}$ Poorer outcome has been observed in patients of rapidly progressing pneumoconiosis who develop pneumothorax. ${ }^{9}$ Pneumoconiosis might be a preexisting lung aetiology for secondary spontaneous pneumothorax ${ }^{10}$; however, the 
incidence of pneumothorax in pneumoconiosis patients is not well established, nor has its risk in pneumoconiosis patients been comprehensively compared with that of the general population. This nationwide population-based cohort study was, therefore, developed using the Taiwan National Health Insurance Research Database (NHIRD) to explore the association between pneumothorax and pneumoconiosis.

\section{METHODS}

\section{Data sources}

The Taiwan NHIRD, compiled and maintained by the Taiwan National Health Research Institute, is an administrative database containing reimbursement information from the Taiwan National Health Insurance (NHI) programme, which has enrolled more than $99 \%$ of Taiwan's population covering ambulatory care, hospital inpatient care, dental services and prescription drugs. ${ }^{12}$ The dataset used for this study was the Longitudinal Health Insurance Database 2005, a cohort of 1 million subjects randomly sampled from the beneficiaries in the Taiwan NHI programme in 2005, which was considered a highly representative sample of Taiwan's general population. ${ }^{13-16}$ The dataset contains information of subjects from the start of the NHI programme in March 1995 to the end of 2013.

\section{Study cohorts}

From the database, we selected patients with a diagnosis of pneumoconiosis (ICD-9-CM codes of 500-505) in at least one inpatient or two ambulatory claims (figure 1). The initial pneumoconiosis diagnosis date was the index date for the pneumoconiosis cohort. Patients were excluded if they had washout periods (from enrolment to the index date) or observation periods (from the index date to the end of follow-up) less than a year, were younger than 18 years or older than 90 years on the index date, or with diagnosis of pneumothorax before the index date. The

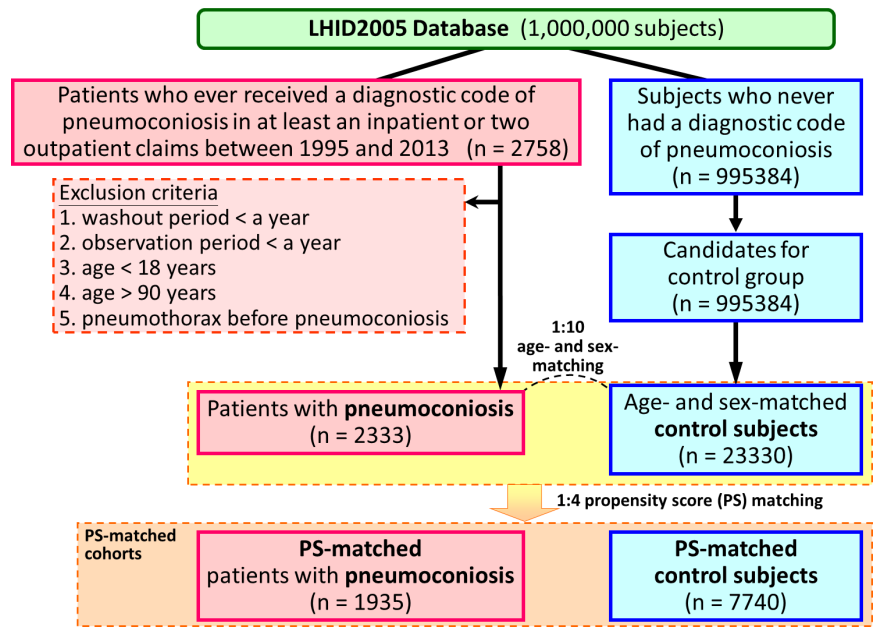

Figure 1 Algorithm for identifying the study population. LHID, Longitudinal Health Insurance Database; PS, propensity score.
ICD-9-CM codes of 512 was used for diagnosis of pneumothorax. For each pneumoconiosis patient, ten agematched and sex-matched control subjects were randomly selected, considering the same exclusion criteria as the pneumoconiosis cohort, and given the same index date as their corresponding pneumoconiosis patient. All the subjects were followed from the index date to either being diagnosed with pneumothorax, end of the study period, withdrawal from the insurance programme or death. To calculate the Charlson Comorbidity Index score, the other comorbidities were also identified by diagnostic codes from the database. ${ }^{17}$ Comorbidities were confirmed with presence of the corresponding diagnostic codes at least twice in the ambulatory database or at least once in the inpatient database before the index date ${ }^{13}$; then, the prevalence, incidence rate (IR) and the cumulative incidence of pneumothorax were compared between pneumoconiosis patients and control subjects.

\section{Statistical analysis}

The categorical variables were described with frequencies and percentages, and the continuous variables were expressed with means and SD, while baseline characteristics were compared between groups by Pearson's $\chi^{2}$ test (for categorical variables) or Student's t-test (for continuous variables). The IR of pneumothorax was calculated as all new cases of pneumothorax during the study period divided by the number of the total personyears, while the IR ratios (IRRs) were estimated by comparing the IRs of pneumothorax in pneumoconiosis patients and control subjects. The 95\% CIs for the IRRs were estimated by Poisson regression. The adjusted IRRs were calculated by multivariable analyses after adjusting for age, sex, residency, income level and the presence of other comorbidities. ${ }^{13-16}$ The Kaplan-Meier method and log-rank test were used to calculate and compare the cumulative incidences of pneumothorax. With adjustment of the same covariates as in Poisson regression, multivariable Cox proportional hazards regression analyses were performed to further assess the effect of pneumoconiosis with the results presented in HRs. Stratified analyses were also performed for Poisson and Cox regression in subgroups of covariates.

Multivariable Cox proportional hazards regression analysis was also used to investigate the risk factor for incident pneumothorax. In addition to the maximal models, reduced multivariable models were developed with backward variable selection method, keeping only variables with $\mathrm{p}<0.05$ from the maximal model.

To account for confounding factors, sex, age, year of the index date, residency, income level and comorbidities were included in a logistic regression model with pneumoconiosis as the dependent variable to determine a propensity score (PS). By selecting four PS-matched control subjects for each pneumoconiosis patient, PS-matched cohorts were extracted from the original cohorts. 
Extraction and computation of data, data linkage, processing and sampling, and statistical analyses were performed using SAS system (V.9.4 for Windows, SAS Institute). The statistical significance level was set at a two-sided $\mathrm{p}<0.05$.

\section{Patient and public involvement}

Data collection was performed from records in the database. There was no increased burden to patients in this study. Results were disseminated alongside increasing awareness in timely caring for pneumoconiosis patients.

\section{RESULTS}

In total, 2333 pneumoconiosis patients from the database were identified and matched with 23330 control subjects by age and sex (figure 1), with table 1 showing

\begin{tabular}{|c|c|c|c|c|}
\hline & All subjects & Pneumoconiosis & Control & $P$ value \\
\hline $\mathrm{N}$ & 25663 & 2333 & 23330 & \\
\hline \multicolumn{5}{|l|}{ Sex, n (\%) } \\
\hline Female & 7447 (29) & $677(29)$ & $6770(29)$ & \\
\hline Male & $18216(71)$ & $1656(71)$ & $16560(71)$ & \\
\hline Age (year), mean $\pm S D$ & $57.4 \pm 15.3$ & $57.4 \pm 15.3$ & $57.4 \pm 15.3$ & \\
\hline \multicolumn{5}{|l|}{ Age (year), n (\%) } \\
\hline$\leq 65$ & $17314(67)$ & $1574(67)$ & $15740(67)$ & \\
\hline$>65$ & $8349(33)$ & $759(33)$ & $7590(33)$ & \\
\hline Residency, n (\%) & & & & $<0.0001$ \\
\hline Northern Taiwan & $7778(30)$ & $1152(49)$ & $6626(28)$ & \\
\hline Other areas & $17885(70)$ & $1181(51)$ & $16704(72)$ & \\
\hline $\begin{array}{l}\text { Monthly income (NT\$), } \\
\text { median (IQR) }\end{array}$ & $\begin{array}{l}19200 \\
(1007-21900)\end{array}$ & $\begin{array}{l}19200 \\
(0-21900)\end{array}$ & $\begin{array}{l}19200 \\
(1007-21900)\end{array}$ & $<0.0001$ \\
\hline Monthly income (NT\$), n (\%) & & & & 0.4691 \\
\hline$\leq 24000$ & $20111(78)$ & $1842(79)$ & $18269(78)$ & \\
\hline$>24000$ & $5552(22)$ & $491(21)$ & $5061(22)$ & \\
\hline $\mathrm{CCl}$ score, mean $\pm \mathrm{SD}$ & $0.7 \pm 1.4$ & $0.8 \pm 1.5$ & $0.7 \pm 1.4$ & $<0.0001$ \\
\hline CCl score, $\mathrm{n}(\%)$ & & & & $<0.0001$ \\
\hline$=0$ & $17740(69)$ & $1408(60)$ & $16332(70)$ & \\
\hline$=1$ & $3777(15)$ & $459(20)$ & $3318(14)$ & \\
\hline$\geq 2$ & $4146(16)$ & $466(20)$ & $3680(16)$ & \\
\hline \multicolumn{5}{|l|}{ Underlying diseases, n (\%) } \\
\hline Heart disease & $692(3)$ & $79(3)$ & $613(3)$ & 0.0310 \\
\hline Myocardial infarction & $224(1)$ & $16(1)$ & $208(1)$ & 0.3084 \\
\hline Congestive heart failure & $505(2)$ & $63(3)$ & $442(2)$ & 0.0075 \\
\hline Peripheral vascular disease & $188(1)$ & $18(1)$ & $170(1)$ & 0.8169 \\
\hline Major neurological disorder & $1814(7)$ & $152(7)$ & $1662(7)$ & 0.2741 \\
\hline Cerebral vascular disease & $1725(7)$ & $146(6)$ & $1579(7)$ & 0.3482 \\
\hline Dementia & $176(1)$ & $12(1)$ & $164(1)$ & 0.2926 \\
\hline Hemiplegia & $202(1)$ & $21(1)$ & $181(1)$ & 0.5171 \\
\hline Chronic pulmonary disease & $3544(14)$ & $619(27)$ & $2925(13)$ & $<0.0001$ \\
\hline Connective tissue disease & $220(1)$ & $17(1)$ & $203(1)$ & 0.4798 \\
\hline Peptic ulcer disease & $2782(11)$ & $276(12)$ & $2506(11)$ & 0.1068 \\
\hline Liver disease & $1731(7)$ & $162(7)$ & $1569(7)$ & 0.6881 \\
\hline Diabetes mellitus & $1765(7)$ & $143(6)$ & $1622(7)$ & 0.1342 \\
\hline Renal disease & $583(2)$ & $46(2)$ & $537(2)$ & 0.3077 \\
\hline Cancer & $809(3)$ & $113(5)$ & $696(3)$ & $<0.0001$ \\
\hline
\end{tabular}

NT\$, New Taiwan Dollar; CCI, Charlson Comorbidity Index; . 
the baseline characteristics of the study population. Patients with pneumoconiosis had more comorbidities than the control subjects, particularly in terms of heart diseases, chronic pulmonary diseases and cancers.

The IR of pneumothorax was significantly higher in pneumoconiosis patients as compared with the subjects in the control cohort ( 1.7 vs 0.5 per 1000 patient-years; adjusted IRR 3.0, 95\% CI 2.7 to 3.3) (table 2). The increased pneumothorax risk related to pneumoconiosis is shown in all strata as stratified by baseline characteristics (table 2). Pneumoconiosis patients, compared with the control subjects, had a significantly higher cumulative incidence of pneumothorax $(\mathrm{p}<0.0001)$ (figure 2A). Stratified analyses revealed that these patients had significantly higher cumulative incidences of pneumothorax than control subjects in female, male, younger and elder subjects (figure 2B-E).

On multivariable Cox regression analysis, pneumoconiosis was an independent risk factor contributing to incident pneumothorax (adjusted HR 3.06, 95\% CI 2.18 to 4.28), $\mathrm{p}<0.0001$ ) (online supplemental table S1). Stratified analyses showed that pneumoconiosis was associated with a higher risk for the development of pneumothorax in all strata (online supplemental figure S1).

Multivariable Cox regression analyses were also used to identify the factors contributing to the development of pneumothorax in the pneumoconiosis patients (table 3). After backward variable selection, it was found that the male sex, heart disease, peripheral vascular disease, chronic pulmonary disease and connective tissue disease were independent risk factors for incidents of pneumothorax in pneumoconiosis patients.

To better account for confounding factors, another set of analyses were performed using PS-matched cohorts, including 1935 pneumoconiosis patients and 7740 control subjects (online supplemental table S2). In the PS-matched cohorts, no significant differences in the baseline characteristics between pneumoconiosis patients and the control subjects were noted. Pneumoconiosis patients had significantly higher IR of pneumothorax than the controls (1.5 vs 0.5 per 1000 patient-years; IRR 3.1, 95\% CI 2.7 to 3.6), and stratified analyses showed similar findings except in the female subjects (online supplemental table S3). The cumulative incidences of pneumothorax were significantly higher in pneumoconiosis patients than in the control subjects in the whole PS-matched cohorts, as well as in stratified analyses of the male, younger and older subjects (online supplemental figure S2). In stratified analysis of female subjects, both pneumoconiosis patients and control subjects had very low cumulative incidence of pneumothorax, which showed no significant difference. Cox regression analysis showed that pneumoconiosis was a risk factor for pneumothorax (HR 3.10, 95\% CI 2.01 to $4.77, \mathrm{p}<0.0001$ ) (online supplemental figure S3). Stratified analyses showed similar results, but the effect of pneumoconiosis on pneumothorax was not found in female subjects.

\section{DISCUSSION}

This is the first long-term nationwide populationbased cohort study to comprehensively explore the associations between pneumoconiosis and pneumothorax. Both incidence and cumulative incidence of pneumothorax were significantly higher in pneumoconiosis patients than in those without pneumoconiosis. In multivariable Cox regression analysis, pneumoconiosis was an independent risk factor for developing pneumothorax after adjusting for age, sex, residency, income levels and other comorbidities. The PS-matched cohort showed similar results in all strata except for female subjects.

Secondary spontaneous pneumothorax has been discussed mostly concerning silicosis. A study observing 50 silicosis patients in India reported a $44 \%$ incident rate of pneumothorax, mainly with unilateral involvement. ${ }^{18}$ A significant association between secondary spontaneous pneumothorax and bullae in acute and accelerated silicosis was found in stone workers in Iran. ${ }^{19}$ The association between silica exposure and emphysema has been noted. ${ }^{20}$ The effect of inflammatory response products on the elastic fibres of the alveolar walls and the coalescence of peri-nodular emphysematous regions were considered to cause the formation of blebs. ${ }^{192122}$ An uneven expansion of the lung due to increased elastic recoil and collapse of adjacent regions following massive fibrosis develops the pressure gradient subsequently to facilitate rupture of the blebs. ${ }^{22} 23$ Piezoelectricity, a phenomenon where crystals generate electronic charges with mechanical stress, might also play some role in the pathophysiology of developing pneumothorax in silicosis due to the free oxygen radicals on the silica crystal surface. ${ }^{1824}$ For other types of pneumoconiosis sharing the traits of inflammatory and fibrotic response, bullae and emphysema are also commonly observed in secondary spontaneous pneumothorax in a progressive state of coal workers' pneumoconiosis $^{25}$; an association between emphysema and asbestosis has also been suggested by epidemiological study of constructors in Canada and Finland..$^{26} \mathrm{~A}$ similar mechanism for silicosis to develop pneumothorax might be applied to most pneumoconiosis patients, though future studies are obligated to investigate the association of pneumothorax in different types of pneumoconiosis.

In this study, the association between pneumoconiosis and pneumothorax in female was not significant, which might be attributed to the small sample size of female subjects. Consistent with other studies investigating the association between pneumothorax and pneumoconiosis, the numbers of female patients were small because dust-exposed occupations are mostly taken by males. An increased prevalence of secondary spontaneous pneumothorax has been reported in silicosis with smoking patients. ${ }^{18} 2428$ In the literature, most patients of pneumoconiosis reported as 


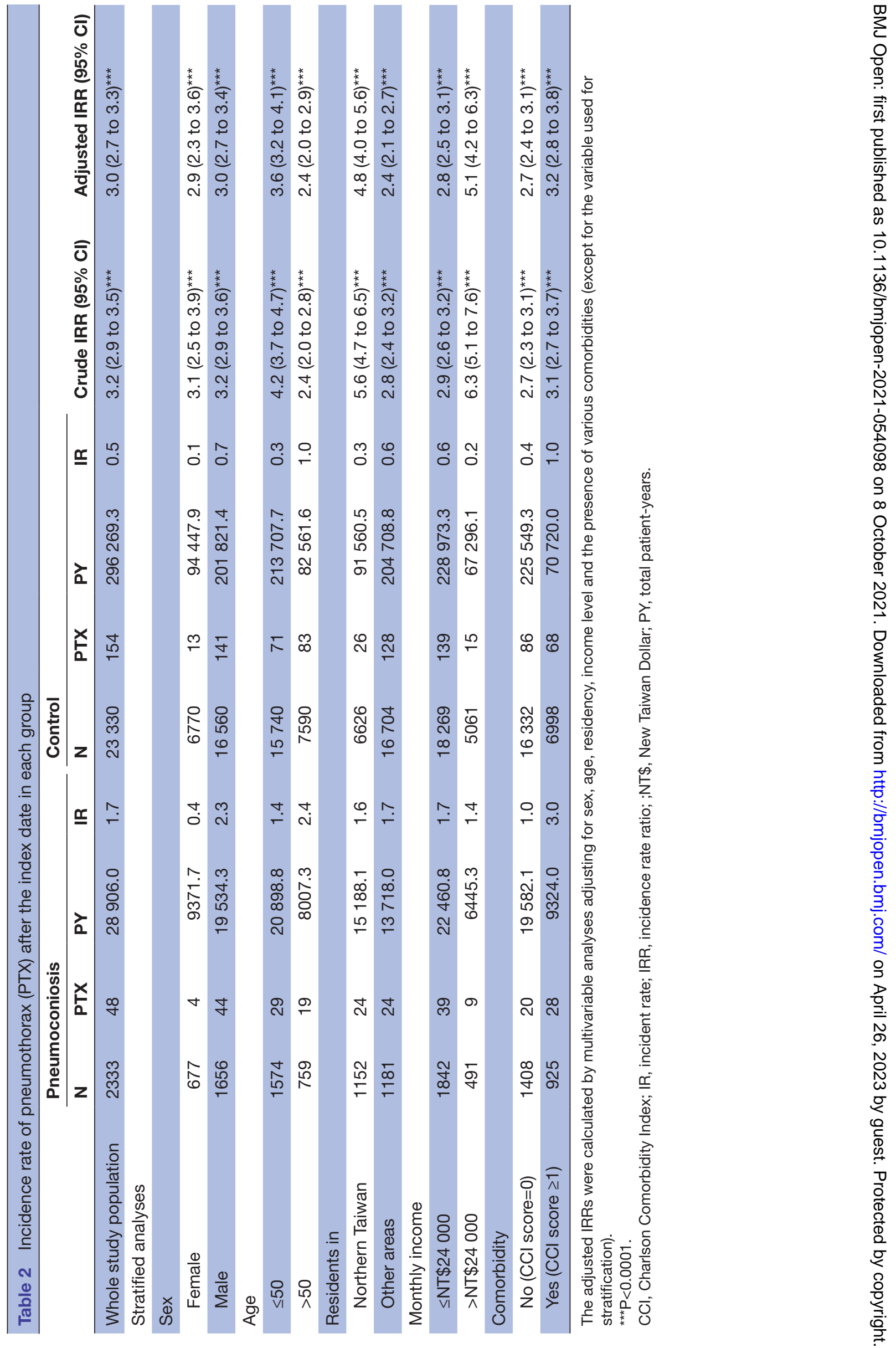



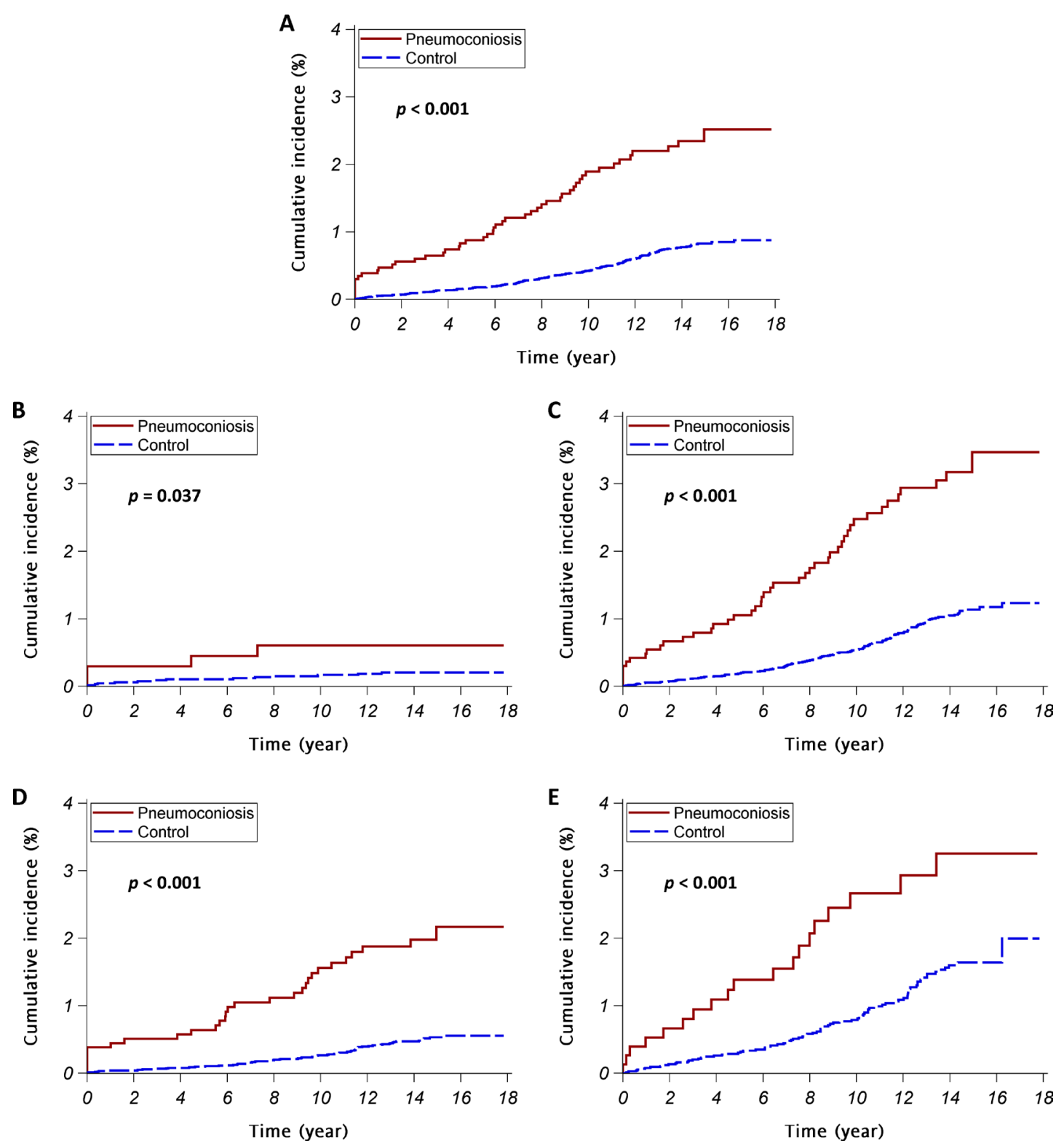

Figure 2 The cumulative incidences of pneumothorax. The red continuous lines and blue dashed lines show the cumulative incidence of pneumothorax for the pneumoconiosis patients and the control subjects, respectively. (A) All study subjects; (B) female subjects; (C) male subjects; (D) subjects aged $\leq 65$ years; (E) subjects aged $>65$ years.

developing pneumothorax had a smoking history (online supplemental table S4). In Taiwan, the prevalence of smoking is markedly lower in females than males, and this could explain why the risk of pneumothorax was increased in female pneumoconiosis patients in our study. However, silicosis per se, in the absence of smoking, might contribute to the development of emphysema, a common underlying cause for secondary spontaneous pneumothorax. ${ }^{19}{ }^{26}$ Without a smoking history recorded in the NHI database, the effect of smoking on the risk of development of pneumothorax in pneumoconiosis patients remains undetermined.

In the current study, being male, having heart disease, peripheral vascular disease, chronic pulmonary disease and connective tissue disease were found as independent risk factors for developing pneumothorax in pneumoconiosis patients. As a major risk factor for pneumothorax, smoking might be an important confounder because the smoking prevalence is much higher in men and smoking is associated with various systemic diseases, including heart disease, peripheral vascular disease and chronic pulmonary disease. Further study adjusting for smoking history is required to evaluate the confounding effect of smoking; nevertheless, it is still clinically valuable to identify the pneumoconiosis patients at higher risk of development of pneumothorax as shown in the current studies.

Secondary pneumothorax in silicosis has often featured with localised pneumothorax due to pleural adhesion, shortly progressing to respiratory failure due to poor 
Table 3 Multivariable Cox regression analysis of the factors contributing to pneumothorax in the pneumoconiosis patients

\begin{tabular}{|c|c|c|}
\hline Variables & Maximal model & Reduced model \\
\hline Male vs female & $4.71(1.67 \text { to } 13.3)^{\star \star}$ & $4.84(1.73 \text { to } 13.57)^{\star *}$ \\
\hline Age $>65$ vs $\leq 65$ & $1.15(0.60$ to 2.24$)$ & \\
\hline Higher income (>NT\$24 000) vs lower income $(\leq \mathrm{NT} \$ 24000)$ & $0.87(0.40$ to 1.90$)$ & \\
\hline \multicolumn{3}{|l|}{ Presence of underlying diseases } \\
\hline Peripheral vascular disease & $11.31(2.48 \text { to } 51.53)^{\star *}$ & $6.76(1.59 \text { to } 28.77)^{\star *}$ \\
\hline Major neurological disorder & $1.32(0.48$ to 3.58$)$ & \\
\hline Chronic pulmonary disease & $2.11(1.12 \text { to } 3.96)^{*}$ & $2.22(1.20 \text { to } 4.09)^{*}$ \\
\hline Connective tissue disease & $7.65(1.73 \text { to } 33.94)^{\star \star}$ & $7.40(1.75 \text { to } 31.24)^{\star \star}$ \\
\hline Peptic ulcer disease & $1.13(0.47$ to 2.70$)$ & \\
\hline Renal disease & $1.25(0.27$ to 5.82$)$ & \\
\hline Cancer & $1.47(0.42$ to 5.22$)$ & \\
\hline
\end{tabular}

The adjusted $\mathrm{HR}$ with $95 \% \mathrm{Cl}$ are presented.

Reduced multivariable models was developed with backward variable selection method, keeping only variables with $p<0.05$, from the maximal model.

${ }^{*} \mathrm{p}<0.05 ;{ }^{* *} \mathrm{p}<0.01$.

NT\$, New Taiwan Dollar.

respiratory functional status with a small amount of pneumothorax, and combined with persistent air leak or refractory pneumothorax. ${ }^{25} \mathrm{~A}$ recurrence rate of spontaneous pneumothorax, as high as $48 \%$, has also been revealed in China in a clinical analysis of 350 pneumoconiosis patients. ${ }^{29}$ The decline in forced expiratory volume in the first second and forced vital capacity values in spirometry were found associated with the occurrence of pneumothorax in rapidly formed silicosis. ${ }^{9}$ As compared with nature stone-associated silicosis, a higher incidence of spontaneous pneumothorax has also been reported in artificial stone-associated silicosis (16.7\% vs $1.6 \%$, $\mathrm{p}<0.05$ ), as well as a more accelerated loss of pulmonary function. ${ }^{30}$

Although the association of spirometry impairment and developing pneumothorax in other types of pneumoconiosis is unclear, a pulmonary function test might be considered during regular follow-up to predict the (poor) outcome. Few studies have focused on management of secondary spontaneous pneumothorax in pneumoconiosis patients. Secondary spontaneous pneumothorax often occurs in the late course of pneumoconiosis along with poor physical and respiratory status, when surgical management is usually contraindicated.$^{31}$ Chemical pleurodesis may be required after chest tube drainage for a persistent air leak or refractory pneumothorax. ${ }^{32}$ Bronchial occlusion with endobronchial Watanabe spigots has also been reported to stop or reduce air leaks effectively. ${ }^{33}$ If surgical intervention is applicable, an absorbable polyglycolic acid patch as a reinforcement or repair material might be helpful to reduce postoperative air leakage for refractory pneumothorax due to silicosis. ${ }^{34}$

With the nationwide, population-based data, this cohort study minimised selection bias and provided a broad view of real-world epidemiology, and with its long follow-up period, has provided better evidence about the temporal trajectory for the association between pneumoconiosis and pneumothorax. However, it is limited by the shortage of clinical information in the NHI database, as potentially important risk factors for pneumothorax such as smoking history, body mass index and family history were unavailable. In the current study, efforts were made to minimise the effect of these confounders by multivariable regression analyses adjusting for baseline characteristics and comorbidities as well as by PS matching. In addition, without information of chest radiography and occupational history, the accuracy of identifying patients with diagnostic codes could not be assessed. Information concerning exposure of dust types, intensity and tenure was also unavailable for more detailed analysis. Future studies with more detailed clinical information are warranted to disclose the effects of these factors.

\section{CONCLUSION}

This large nationwide population-based cohort study confirmed that patients of pneumoconiosis had significantly higher risk of developing pneumothorax compared with matched control subjects without pneumoconiosis. 
Clinicians should be aware of the risk of pneumothorax in such patients, especially in males with heart disease, peripheral vascular disease, chronic pulmonary disease and connective tissue disease.

\section{Author affiliations}

${ }^{1}$ Department of Occupational and Environmental Medicine, Kaohsiung Medical University Hospital, Kaohsiung Medical University, Kaohsiung, Taiwan

${ }^{2}$ Graduate Institute of Medicine, College of Medicine, Kaohsiung Medical University, Kaohsiung, Taiwan

${ }^{3}$ Division of Pulmonary and Critical Care Medicine, Department of Internal Medicine, Kaohsiung Medical University Hospital, Kaohsiung Medical University, Kaohsiung, Taiwan

${ }^{4}$ School of Medicine, College of Medicine, Kaohsiung Medical University, Kaohsiung, Taiwan

${ }^{5}$ Department of Respiratory Care, College of Medicine, Kaohsiung Medical University, Kaohsiung, Taiwan

${ }^{6}$ Department of Internal Medicine, Kaohsiung Municipal Ta-Tung Hospital, Kaohsiung Medical University, Kaohsiung, Taiwan

Acknowledgements We thank Tse-Kuang Kai for assistance in statistical analysis. The authors also thank the Statistical Analysis Laboratory, Department of Internal Medicine and the Statistical Analysis Laboratory, Department of Medical Research, Kaohsiung Medical University Hospital for their help.

Contributors J-HP, C-HC, C-LW, C-YD, C-CS, M-JT, J-YH and I-WC contribute to the conceptualisation of the study. J-HP, C-HC and M-JT were responsible for data collection and analysis. J-HP and C-HC draft the manuscript. M-JT and J-YH extensively revised the manuscript. All authors reviewed and approved the final manuscript.

Funding This work was supported by grants from Kaohsiung Medical University Hospital (grant No.: KMUH107-7M37) and Kaohsiung Medical University (grant No.: KMU-Q108005).

Competing interests None declared.

Patient consent for publication Not applicable.

Ethics approval This study was approved by the Kaohsiung Medical University Hospital Institutional Review Board (KMUHIRB-EXEMPT(II)-20150065 and KMUHIRB-EXEMPT-20190081). Data were anonymised before the authors accessed them for the purpose of this study.

Provenance and peer review Not commissioned; externally peer reviewed.

Data availability statement This study is based on data from the National Health Insurance Research Database provided by the National Health Insurance Administration, Ministry of Health and Welfare, and managed by the National Health Research Institutes. The interpretation and conclusions contained herein do not represent those of the National Health Insurance Administration, Ministry of Health and Welfare or National Health Research Institutes (NHRI). Due to legal and ethical restrictions, researchers should contact NHRI (https://nhird.nhri.org.tw/) for access of the data after approval by the Institutional Review Board. No data are directly available. This study is based on data from the National Health Insurance Research Database provided by the National Health Insurance Administration, Ministry of Health and Welfare and managed by National Health Research Institutes. The interpretation and conclusions contained herein do not represent those of National Health Insurance Administration, Ministry of Health and Welfare or National Health Research Institutes (NHRI). Due to legal and ethical restrictions, researchers should contact NHRI (http://nhird.nhri.org.tw/index.htm) for access of the data after approved by institutional review board.

Supplemental material This content has been supplied by the author(s). It has not been vetted by BMJ Publishing Group Limited (BMJ) and may not have been peer-reviewed. Any opinions or recommendations discussed are solely those of the author(s) and are not endorsed by BMJ. BMJ disclaims all liability and responsibility arising from any reliance placed on the content. Where the content includes any translated material, BMJ does not warrant the accuracy and reliability of the translations (including but not limited to local regulations, clinical guidelines, terminology, drug names and drug dosages), and is not responsible for any error and/or omissions arising from translation and adaptation or otherwise.

Open access This is an open access article distributed in accordance with the Creative Commons Attribution Non Commercial (CC BY-NC 4.0) license, which permits others to distribute, remix, adapt, build upon this work non-commercially, and license their derivative works on different terms, provided the original work is properly cited, appropriate credit is given, any changes made indicated, and the use is non-commercial. See: http://creativecommons.org/licenses/by-nc/4.0/.

\section{ORCID iDs}

Chau-Chyun Sheu http://orcid.org/0000-0002-7979-3749

Ming-Ju Tsai http://orcid.org/0000-0003-3621-3334

\section{REFERENCES}

1 Driscoll T. Occupational airborne particulates: assessing the environmental burden of disease at national and local levels (2004Environmental burden of disease series, no 7). Geneva: World Health Organization, 2004.

2 Harrison RJ, LaDou J. Current occupational \& environmental medicine. New York: McGraw-Hill, 2014.

3 Fujimura N. Pathology and pathophysiology of pneumoconiosis. Curr Opin Pulm Med 2000;6:140-4.

4 Qi X-M, Luo Y, Song M-Y, et al. Pneumoconiosis: current status and future prospects. Chin Med J 2021;134:898-907.

5 Zosky GR, Hoy RF, Silverstone EJ, et al. Coal workers' pneumoconiosis: an Australian perspective. Med J Aust 2016;204:414-8.

6 Blackley DJ, Halldin CN, Laney AS. Continued increase in prevalence of coal workers' pneumoconiosis in the United States, 1970-2017. Am J Public Health 2018;108:1220-2.

7 GBD 2017 Disease and Injury Incidence and Prevalence Collaborators. Global, regional, and national incidence, prevalence, and years lived with disability for 354 diseases and injuries for 195 countries and territories, 1990-2017: a systematic analysis for the global burden of disease study 2017. Lancet 2018;392:1789-858.

8 GBD 2017 Causes of Death Collaborators. Global, regional, and national age-sex-specific mortality for 282 causes of death in 195 countries and territories, 1980-2017: a systematic analysis for the global burden of disease study 2017. Lancet 2018;392:1736-88.

9 Mohebbi I, Rad IA. Secondary spontaneous pneumothorax in rapidly progressive forms of silicosis: characterization of pulmonary function measurements and clinical patterns. Toxicol Ind Health 2007;23:125-32.

10 Noppen M, De Keukeleire T. Pneumothorax. Respiration 2008;76:121-7.

11 Sahn SA, Heffner JE. Spontaneous pneumothorax. N Engl J Med Overseas Ed 2000;342:868-74.

12 National Health Insurance Research Database - Background. Available: https://nhird.nhri.org.tw/en/index.html [Accessed April, 25th, 2019].

13 Chen C-M, Tsai M-J, Wei P-J, et al. Erectile Dysfunction in Patients with Sleep Apnea--A Nationwide Population-Based Study. PLoS One 2015;10:e0132510.

14 Chen C-M, Kuo C-Y, Wu M-N, et al. Increased risk of major depressive disorder in sleep apnea patients in Taiwan. Sci Rep 2021;11:765.

15 Liu P-K, Chang Y-C, Tai M-H, et al. The association between central serous chorioretinopathy and sleep apnea: a nationwide populationbased study. Retina 2020;40:2034-44.

$16 \mathrm{KL} \mathrm{W}$, Kuo CY, Tsai YC. CHADS, $\mathrm{CHA}_{2} \mathrm{DS}_{2} \mathrm{ASc}$, and new ABCD scores predict the risk of peripheral arterial disease in patients with sleep apnea. J Clin Med 2019;8:188.

17 Deyo RA, Cherkin DC, Ciol MA. Adapting a clinical comorbidity index for use with ICD-9-CM administrative databases. J Clin Epidemiol 1992;45:613-9.

18 Meena MK, Singh R, Joshi N, et al. Silicosis with secondary spontaneous pneumothorax in the Western Rajasthan. Cureus 2020;12:e11811.

19 Mohebbi I, Hassani E, Salarilak S, et al. Do bullae and emphysema increase risk of pneumothorax in silicosis? J Occup Med Toxicol 2007;2:8.

20 Cohen R, Velho V. Update on respiratory disease from coal mine and silica dust. Clin Chest Med 2002;23:811-26.

21 Gupta KB, Manchanda M, Kaur P. Bilateral spontaneous pneumothorax in silicosis. Indian J Chest Dis Allied Sci 2006;48:201-3.

22 Dixit R, Meena M, Patil CB. Pneumomediastinum, bilateral pneumothorax and subcutaneous emphysema complicating acute silicosis. Int J Occup Med Environ Health 2015;28:635-8.

23 MACKLIN MT, Macklin CC. Malignant interstitial emphysema of the lungs and mediastinum as an important occult complication in many respiratory diseases and others conditions: interpretation 
of clinical literature in light of laboratory experiment. Medicine 1944;23:281-358.

24 Bairwa MK, Joshi N, Agnihotri SP. Silicosis with bilateral spontaneous pneumothorax in Rajasthan. Indian J Occup Environ Med 2019;23:112-6.

25 Jun JS, Jung JI, Kim HR, et al. Complications of pneumoconiosis: radiologic overview. Eur J Radiol 2013;82:1819-30.

26 Bégin R, Filion R, Ostiguy G. Emphysema in silica- and asbestosexposed workers seeking compensation. A CT scan study. Chest 1995;108:647-55.

27 Huuskonen O, Kivisaari L, Zitting A, et al. Emphysema findings associated with heavy asbestos-exposure in high resolution computed tomography of Finnish construction workers. J Occup Health 2004;46:266-71.

28 Sharma RK, Luhadia A, Luhadia SK, et al. Pneumothorax in the cases of silicosis in southern part of Rajasthan. International Journal of Advances in Medicine 2017;4:1547.
29 Li Y, Zhang XH, Long ZY, et al. [Clinical analysis of 350 cases of pneumoconiosis complicated with spontaneous pneumothorax]. Zhonghua Lao Dong Wei Sheng Zhi Ye Bing Za Zhi 2020;38:48-50.

30 Wu N, Xue C, Yu S, et al. Artificial stone-associated silicosis in China: a prospective comparison with natural stone-associated silicosis. Respirology 2020;25:518-24.

31 Iga N, Nishi H, Fujimoto N, et al. Clinical features of secondary spontaneous pneumothorax complicated with silicosis. Respir Investig 2018;56:144-9.

32 Kawano M, Miura H, Anan H, et al. Treatment of secondary spontaneous pneumothorax complicating silicosis and progressive massive fibrosis. Kurume Med J 2002;49:35-40.

33 Kaneda H, Minami K-ichiro, Nakano T, et al. Efficacy and long-term clinical outcome of bronchial occlusion with endobronchial Watanabe spigots for persistent air leaks. Respir Investig 2015;53:30-6.

34 Lin X-M, Liu Y, Chi C, et al. Efficacy of an absorbable polyglycolic acid patch in surgery for pneumothorax due to silicosis. $J$ Cardiothorac Surg 2012;7:18. 